\title{
Spectropolarimetry of a sunspot at disk centre
}

\author{
M. Sánchez Cuberes ${ }^{1}$, K. G. Puschmann ${ }^{2}$, and E. Wiehr ${ }^{2}$ \\ 1 Astrophysikalisches Institut Potsdam, An der Sternwarte 16, 14482 Potsdam, Germany \\ e-mail: msanchez@aip.de \\ 2 Universitäts-Sternwarte, Geismarlandstr. 11, 37083 Göttingen, Germany \\ e-mail: [kgp; ewiehr]@uni-sw.gwdg.de
}

Received 21 September 2004 / Accepted 3 February 2005

\begin{abstract}
The magnetic, thermal and velocity structure of a sunspot at the solar disk centre $\left(\theta=2^{\circ}\right)$ is investigated by inverting the full Stokes profiles of three infrared lines. A single magnetic component atmosphere is assumed with height gradients of the physical quantities. Since the line-of-sight (LOS) is perpendicular to the solar surface, differential optical or projection effects do not interfere, as often is the case for the usual observations at oblique LOS. We find a symmetric configuration of the field and flow and the downward motion that increases with radial distance by up to $3 \mathrm{~km} \mathrm{~s}^{-1}$ near the outer penumbral border. The magnetic field is found to be highly axially symmetric without any indication of azimuthal vortices. A tight relation between field strength and inclination is obtained with a gradient of $35^{\circ} / 1000 \mathrm{G}$ independent of height. The penumbra shows "spines" hosting a pronounced negative correlation between field strength and inclination in the sense that steeper and stronger magnetic fields are related to brightenings in the line cores but not in the continuum. We discuss the dependence of the obtained results on different assumptions of parasitic light, and present indications of its overestimation by the inversion code.
\end{abstract}

Key words. Sun: photosphere - Sun: sunspots - techniques: polarimetric - Sun: magnetic fields - line: profiles

\section{Introduction}

Due to their occurrence at latitudes of the solar activity belts, sunspots are mostly seen under a line-of-sight (LOS) not perpendicular to the solar surface. This produces projection effects which can only be considered under certain assumptions about the spot geometry being particularly important when trying to disentangle structures of individual inclination. A finite angle of the LOS with the inclined field and flow causes a sampling of different atmospheric heights in the limb-side and the centreside penumbra, producing an apparent morphological asymmetry between them; there is still controversy as to which side samples deeper layers (Rimmele 1995; Westendorp Plaza et al. 2001a,b).

Under plasma conditions, the gas-flow is expected to follow the field lines. However, the mean magnetic field has a larger inclination to the solar surface than the Evershed flow (see Stellmacher \& Wiehr 1971, and references therein), which is found to be largely horizontal (Wiehr et al. 1984; Balthasar et al. 1996; Westendorp Plaza et al. 2001b). This suggests the existence of (at least) two magnetic field components (Bernasconi et al. 1998; Leka 2001; Bellot Rubio et al. 2004), moreover, arguments were given for bundles of optically thin field components (Sánchez Almeida 1998). At the disk centre, the interlacing of differently inclined spot structures with significant relative Doppler shifts is small, making a determination of e.g. penumbral fluting difficult. However, several properties of the magnetic, the thermal and the flow configuration of sunspots can be optimally studied if the LOS is perpendicular to the solar surface.

Already a few degrees outside the disk centre (Stellmacher $\&$ Wiehr 1980), the flow channels are seen as up-flows in the centre-side and as down-flows in the limb-side penumbra. Bello González et al. (2004) as well as Schlichenmaier et al. (2005) showed that the relation between flow, field, and intensity significantly differs between centre- and limb-side penumbra. Since the spectral lines contain only information about the LOS velocity component, some assumption has to be made to obtain the full velocity vector: this is commonly azimuthal averaging assuming axial symmetry. The small vertical velocity components obtained in this way must be treated with caution since they are deduced from the LOS component, largely dominated by the strong horizontal flow. Any deviation from the axial symmetry will strongly affect the retrieved values. A determination of the flow angle assuming axial symmetry is particularly problematic at the spot border, just where the deduced vertical velocity values become significant (Schlichenmaier \& Schmidt 2000). This method may then be reliable to determine the strong horizontal flow component, but the small vertical and azimuthal velocity components should be treated with caution (Solanki 2003).

Here, we study the thermal, flow and magnetic configuration of a symmetrical sunspot at a heliocentric angle of $2^{\circ}$. 
Table 1. Atomic parameters of the three lines used for polarimetry; $g_{\text {eff }}=$ effective Landé factor, $\chi_{\text {low }}=$ lower excitation potential, $g=$ statistical weight, $f=$ oscillatory strength.

\begin{tabular}{lcccc}
\hline \hline Element & $\lambda[\AA]$ & $g_{\text {eff }}$ & $\chi_{\text {low }}[\mathrm{eV}]$ & $\log (g f)$ \\
\hline Fe I & 10783.040 & 1.5 & 3.111 & -2.712 \\
Si I & 10784.551 & 0.92 & 5.964 & -0.593 \\
Si I & 10786.851 & 1.5 & 4.930 & -0.305 \\
\hline
\end{tabular}

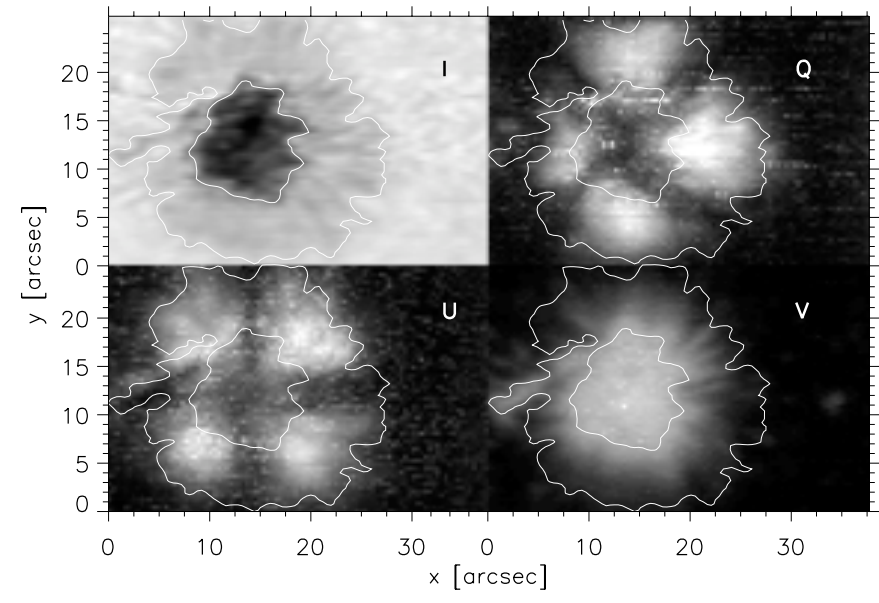

Fig. 1. Maps of the disk centre sunspot from Sep. 23, 2003, showing spectrally integrated Stokes- $I,-Q,-U$, and $-V$ absolute values.

\section{Observations and data analysis}

On 23 September, 2003, a largely symmetric sunspot passed the central meridian at a solar latitude of $9^{\circ} \mathrm{N}$, almost perfectly compensated by the inclination of the solar axis $B_{0}=7^{\circ}$. We observed this sunspot at a heliocentric angle of $\theta \sim 2^{\circ}$ (i.e. $\mu=0.9994)$ with the Tenerife Infrared Polarimeter (TIP; Martínez Pillet et al. 1999) at the $\oslash=70 \mathrm{~cm}$ Vacuum Tower Telescope (VTT) on Tenerife. We took the full Stokes vector through the line profiles of Fe I $10783 \AA$, Si I $10784 \AA$, Si I $10787 \AA$ (Table 1 ; note that the Si I $10784 \AA$ line splits in a more complex pattern than a normal Zeeman triplet). The spatial sampling was 0.4 ; the VTT correlation tracker largely compensated for image motion. The Stokes spectra were dark subtracted and flat-fielded in the usual manner. Instrumental polarisation was corrected following the statistical method described in Schlichenmaier \& Collados (2000). The spectral resolution finally achieved with TIP and its detector is $32 \mathrm{~m} \AA$. An exposure time of $50 \mathrm{~ms}$ for each single slit position was used to scan the spot in 64 steps within $370 \mathrm{~s}$.

The spatial distribution of the integrated Stokes- $I$ and - $V$ profiles (Fig. 1) shows a conspicuously high axial symmetry with respect to the sunspot centre: Stokes- $V$ decreases radially, Stokes- $Q$ and $-U$ increase with distance from the spot centre and exhibit an azimuthally varying $U / Q$ ratio which indicates a high symmetry of the horizontal field component. The Stokes- $I$ image shows that the spatial resolution is just sufficient to resolve some penumbral structures.

We do not find "complex"- $V$ profiles inside the sunspot; only in a ring around the sunspot do locations with anomalous (i.e., 3-lobed) Stokes- $V$ profiles occur. We attribute this to the purely vertical view through the largely horizontal flow channels which barely yields a radiative interlace of fine-structure atmospheres with significant relative Doppler shifts. The Stokes- $V$ profiles show area asymmetries $\left(\delta A=\int V(\lambda) \mathrm{d} \lambda / \int|V(\lambda)| \mathrm{d} \lambda\right)$ smaller than $1 \%$ that are probably due to the smaller sensitivity of infrared lines to discontinuities along the LOS (cf., Borrero et al. 2004) and to the disk centre position of our spot.

\subsection{Sensitivity of the lines}

The characteristic parameters of the three lines used are given in Table 1. Calculations of their response functions (RF) for temperature, LOS-velocity and magnetic field are shown in Fig. 2 for model atmospheres of the quiet sun, mean penumbra and umbra. The three lines span a height range sufficiently large for a deduction of the vertical gradient at all positions in the sunspot map. The sensitivity of the lines and their height variation to the different relevant physical quantities are presented in Fig. 3 as 1-D cuts of the RFs at different line positions. They show that the lines used are sensitive in a range from $\log \tau=0.5$ up to $\log \tau=-2.5$; however, we restrict our results to the region $0>\log \tau>-2$. The optical depths $\tau$ refer to the standard wavelength $\lambda=5000 \AA\left(\tau=\tau_{5000}\right)$.

Comparison with the formation height ranges of the often used lines Fe I $6301.5 \AA$, Fe II $6302.5 \AA$, Fe I $15648.5 \AA$ and Fe I $15652.8 \AA$ shows that the lines used in this work are formed at similar layers as Fe I 6301.5 $\AA$ and Fe II 6302.5 but at layers higher than the lines in the $1.5 \mu \mathrm{m}$ range. The different results obtained by inversion of sunspot spectra in these two spectral ranges have often been attributed to differences in the formation heights of infrared and visible lines (Mathew et al. 2003; Borrero et al. 2004). The lines used in the present paper are sampling the same layers as visible lines but with the "IR sensitivity" to magnetic fields (and other physical quantities).

\subsection{Inversion procedure}

The reduced profiles have been inverted by means of the SIR code (Stokes Inversion based on Response functions; Ruiz Cobo \& del Toro Iniesta 1992). This code returns for each pixel the depth stratification of temperature $(T)$, magnetic field strength $(B)$, field inclination $(\gamma)$, field azimuth $(\phi)$, LOS velocity $(v)$ and single values for micro- and macro-turbulence, both assumed to be constant with depth.

We find that a two-component inversion yields an almost zero filling factor for one of the magnetic components throughout most of the sunspot area. Numerical tests performed by Borrero et al. (2004) show that two-component inversions present difficulties in reproducing two magnetic atmospheres which produce simple double-lobed Stokes- $V$ profiles. Bellot Rubio (2003) demonstrated that one component inversions with gradients of the physical quantities are able to properly describe the general structure of sunspots, including 

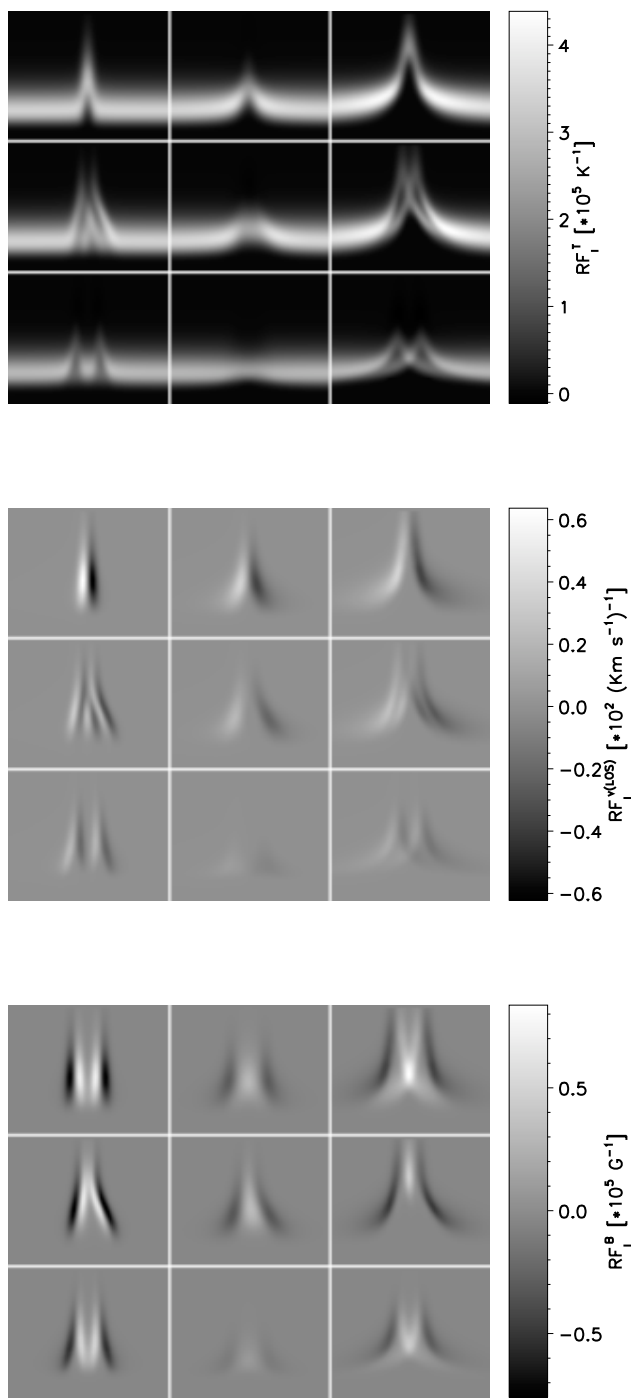
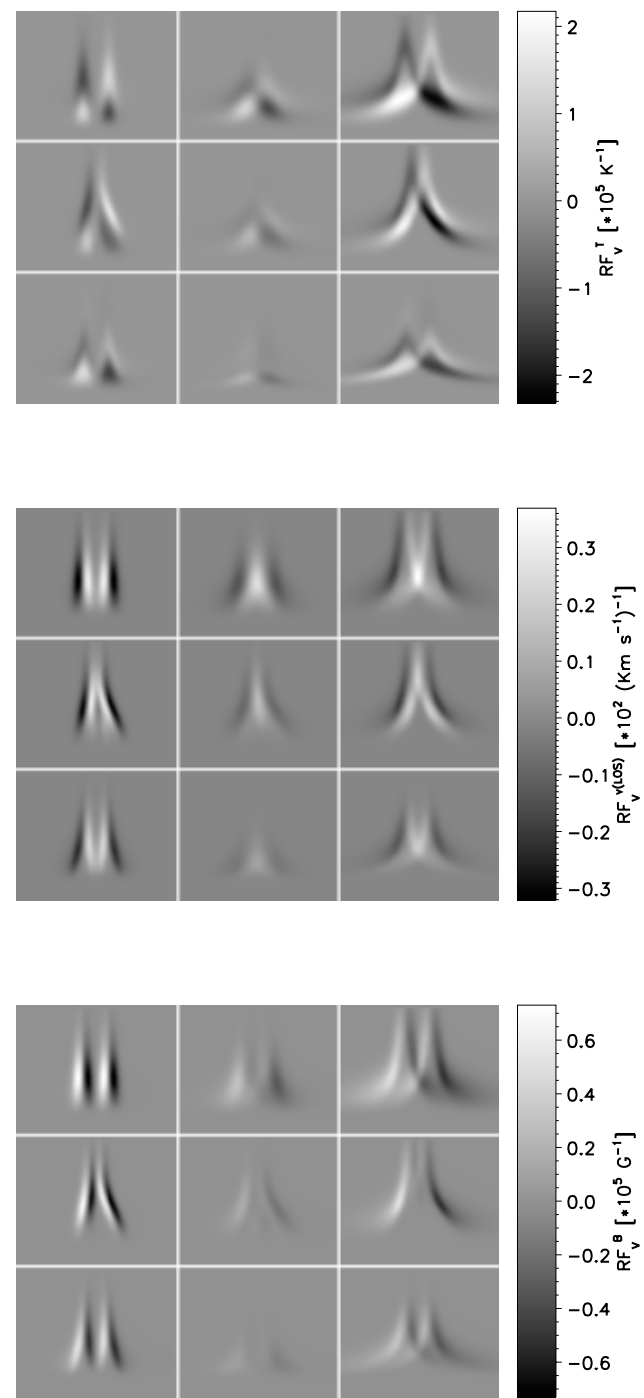

Fig. 2. Response of Stokes I (left panels) and V (right panels) to temperature (upper panels), velocity (middle panels) and magnetic field (lower panels) for Fe I $10783 \AA$ (left columns), Si I $10784.5 \AA$ (middle columns), and Si I $10786.8 \AA$ (right columns) for a quiet Sun atmosphere (upper rows), mean penumbral atmosphere (middle rows) and hot umbral atmosphere (lower rows); each subpanel spans ordinates from log $\tau=1.2$ to $\log \tau=-4$ and abscissa of $2 \AA$.

asymmetries often observed in the Stokes profiles. We thus perform an inversion with a single magnetic component.

The height range spanned by the three lines allows the inversion code to determine vertical gradients of the various physical quantities, except for the macro-turbulence and the non-thermal line broadening parameter "micro-turbulence", both set to be constant with height. We removed the $180^{\circ}$ ambiguity from the transverse Zeeman effect, assuming a smooth variation of the magnetic field vector. The disk centre position of the sunspot yields the magnetic field inclination directly as the zenith angle, i.e., no conversion to a local reference frame is required.

\subsection{Parasitic light}

The SIR code is able to consider "scattered light" allowing a certain amount of the Stokes- $I$ profile to be of non-local origin, i.e. to stem from the neighbourhood of the location under study. This "parasitic" line profile may be photospheric or even magnetically split, however, polarisation profiles cannot be considered by SIR. The code is also unable to distinguish real "straylight" from intrusions of field-free material into one resolution element; an assumed straylight factor of $\alpha$ is equivalent to a magnetic filling factor of $1-\alpha$. Unsplit line profiles from the quiet photosphere can either originate from scattering, image motion or blurring.

Scattering brings light from the entire solar disk into the sunspot; it depends almost exclusively on the state of the optical surfaces (Stellmacher \& Wiehr 1970); at the VTT, only the coelostat mirrors and the entrance window may be covered by dust. Since the scattering decreases with wavelength with a power of -1.9 (Wöhl et al. 1970), in the infrared spectral region it is $1 / 4$ of that in the visible range and thus almost negligible. Image motion moves the spot image as a whole over the spectrograph slit and is in our spectra largely suppressed by the use of the VTT correlation tracker (tip-tilt mirror). Blurring 

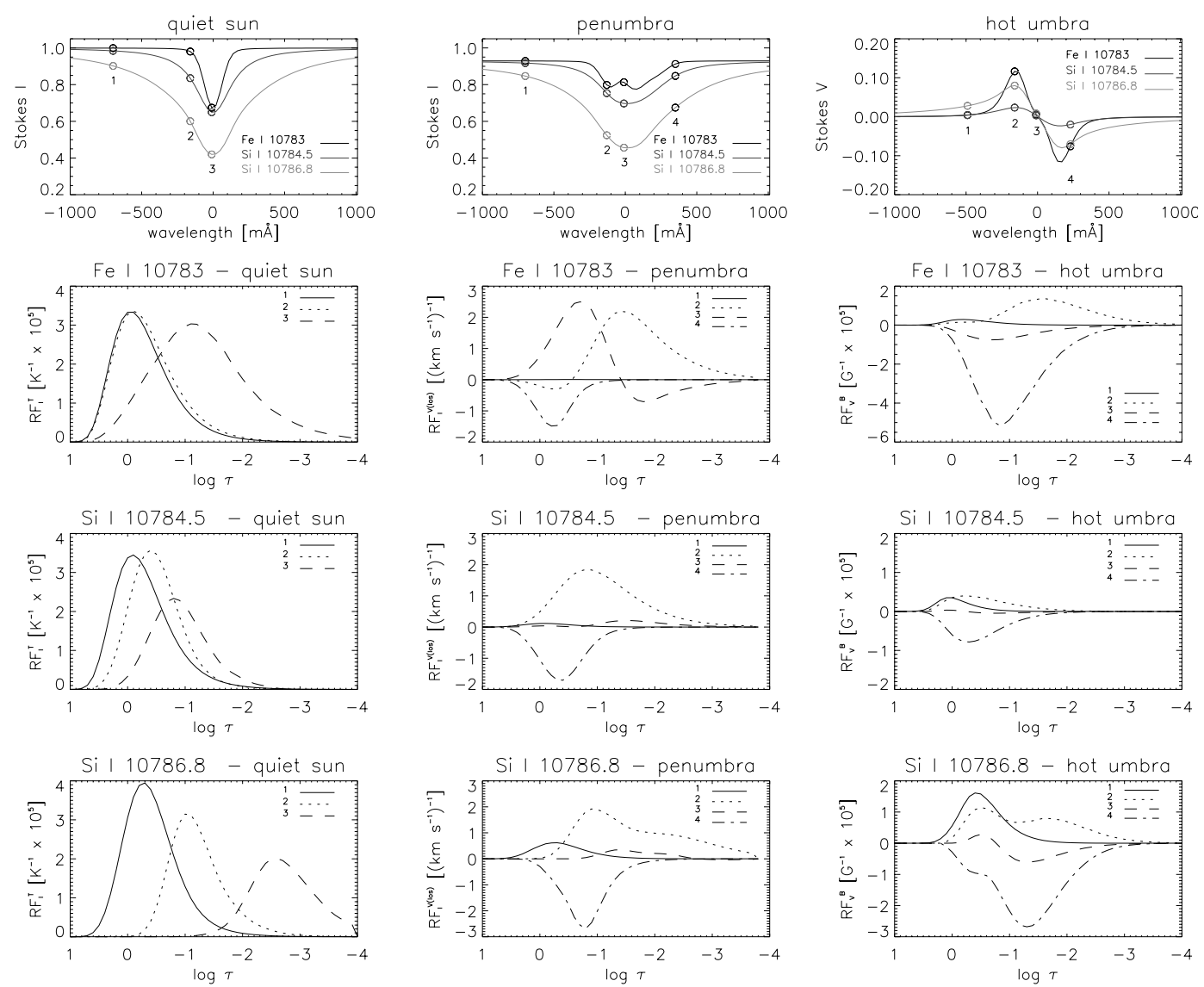

Fig. 3. Response functions (RF) of Stokes-I to temperature in a quiet sun atmosphere (left panels), to LOS velocity in a penumbral atmosphere (middle panels) and of Stokes- $V$ to magnetic field in a hot umbral atmosphere (right panels) at different positions throughout the three lines observed.

could have been minimised by adaptive optics but this was not available at the time.

Hence, we consider blurring as the main source of parasitic light in our spectra. It affects any location of the (rather small) spot with light from its vicinity: the outer penumbra by light from the surrounding photosphere, the inner penumbra by polarised light from the outer penumbra (from the umbra much less since it is darker), and the umbra by Stokes profiles from the inner penumbra. Blurring may well act over some arcsec distance as visible in the inner penumbral splitting of the $\mathrm{Fe}^{+} 6149$ line even throughout the umbra (Stellmacher \& Wiehr 1970, their Fig. 3). However, the quality of our images showing a sharp outer penumbral edge and visible granulation, indicates that blurring does not exceed a few arcsec.

A pollution of sunspot spectra by polarisation profiles has so far not been treated; also in the SIR-code only "parasitic" Stokes- $I$ profiles are considered. If these are of photospheric origin, they maximally amount to a few percent (see above). We thus consider $5 \%$ as upper limit; tests with $0 \%$ parasitic light in our spectra do not lead to essential differences. However, if the "parasitic" light is automatically fitted by the SIR-code as a free parameter, it amounts for our spectra to $5 \%-10 \%$ in the umbra and to $30 \%$ in the inner penumbra, increasing at its outer border to $50 \%-60 \%$ of the apparent local intensity. These values are in agreement with those obtained by similar inversions
(Westendorp Plaza et al. 1997; Mathew et al. 2003); however, they are far from our realistic estimate of blurring given above. Similarly, Martinez Pillet (1992) suggests a small influence of parasitic light.

The large amount of "straylight" automatically fitted by the SIR-code cannot be interpreted as intrusions of field-free material, since Hewagama et al. (1993) estimate an upper limit of $10 \%-20 \%$ in the outer and $5 \%$ in the inner penumbra. Degenhardt \& Lites (1993) show that such field-free intrusions would be observable only in layers deeper than those sampled by the lines used here. Schmidt \& Balthasar (1994) gave further evidence that such field-free intrusions indeed do not occur.

In the present paper, we discuss our results separately for a constant (5\%) and a SIR-adopted "straylight".

\section{Results and discussion}

The SIR code returns a height variation of the various physical quantities for each spatial location which allows us to construct two-dimensional maps for each height level. The smooth spatial variations obtained prove the quality of our inversion. The largely roundish sunspot allows us to determine azimuthal variations of the various parameters along circular azimuthal paths (Fig. 4). In order to additionally determine mean radial variations, we average the respective parameters along concentric circles around the sunspot centre, and plot these "azimuthal 


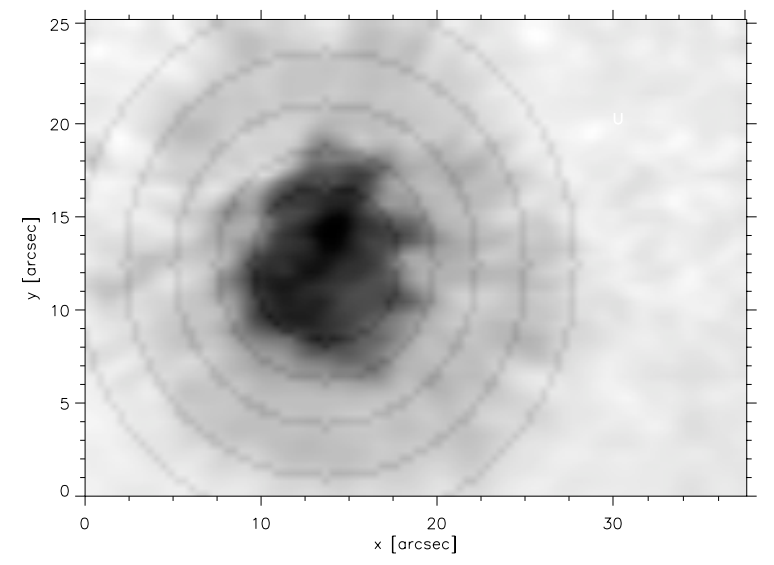

Fig. 4. Circular paths selected to determine azimuthal means of the deduced parameters.

means" as a function of the radial distance. The results are given in the respective subsections; selected azimuthal scans are presented in Sect. 3.4.

\subsection{Thermal configuration}

The interpretation of split line profiles and their polarisation is not an optimal tool for the determination of the temperature stratifications in umbra and penumbra; this is much better done with non-magnetic lines (Stellmacher \& Wiehr 1970). However, our inversion yields this stratification for each location in the sunspot as a "by-product", so that we also present these results. The dark umbral core and the mean penumbral models are given in Fig. 5 together with stratifications published by other authors.

The Stokes- $I$ data yield a mean umbral intensity of $I^{\text {umbra }}=$ $0.52 \times I^{\text {phot }}$ which, according to SIR, corresponds to a temperature of $4800 \mathrm{~K}$ at $\log \tau_{5000}=0$ (Fig. 5). The darkest umbral core has $I^{\text {umbra }}=0.47 \times I^{\text {phot }}$, indicating an intrinsically hotter umbral background compared to predictions of cool umbral models (e.g., Stellmacher \& Wiehr 1981) which give $I^{\text {umbra }}=0.34 \times I^{\text {phot }}$ for $1.1 \mu \mathrm{m}$. The "hotter" umbrae cannot be explained by a large population of dots; this would produce a blue continuum excess which is not observed (Wiehr \& Stellmacher 1984). Brighter umbrae must thus have an intrinsically hotter inter-dot "background", which can be modelled neither with up-scaled atmospheres of darker umbrae, nor with down-scaled photospheric models such as, e.g. the one by Mathew et al. (2003) in Fig. 5.

For the mean penumbra, we observe continuum contrasts of $I^{\text {penumbra }}=0.93 \times I^{\text {phot }}$ in agreement with Schmidt \& Fritz (2004), who found $I^{\text {penumbra }}=0.90 \times I^{\text {phot }}$. The penumbral temperature stratification obtained from our data is thus close to models in the literature (Fig. 5). We find an azimuthally symmetrical temperature distribution in the penumbra which justifies an azimuthal averaging. In contrast, previous observations found asymmetrically distributed intensities (and thus temperatures) from data in centre- and limb-side penumbrae outside the disk centre (Tritschler et al. 2004; Bello Gonzalez et al. 2004).
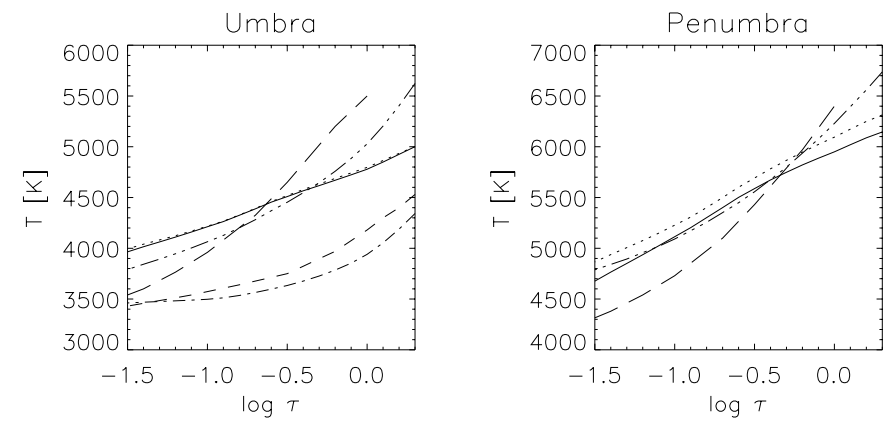

Fig. 5. Temperature as a function of $\log \tau_{5000}$ for umbra (left) and mean penumbra (right panel). Solid lines: darkest umbral core with SIR-adapted straylight; dotted lines: with a constant straylight of 5\%; long-dashes: Mathew et al. (2003); short dashes (left panel): cold umbral model by Stellmacher \& Wiehr (1981); dot-dashes (left panel: cold umbral model by Collados et al. (1994); three-dot-dashes (left panel): hot umbral model by Collados et al. (1994); three-dot-dashes (right panel): mean penumbral model by del Toro Iniesta et al. (1994).

An interesting feature in sunspots is a "bright rim" in the inner penumbra that has been observationally well established (Rimmele 1995; Balasubramaniam 2002; Bellot Rubio 2003; Tritschler et al. 2004). Azimuthally-averaged intensities, given in Fig. 6 as a function of the radial distance from the spot centre, show that a pronounced relative intensity enhancement occurs in the line cores but not in the line wings nor in the continuum. As a consequence, the radial variation of the temperature, returned by SIR, shows for the inner penumbra a local temperature enhancement for the high layers. Besides, it occurs only if a constant straylight of 5\% is considered; if the "straylight" is adapted by SIR, that temperature hump is more pronounced, and additionally occurs in deep layers (Fig. 6). This shows that the deduced temperatures are highly sensitive to the assumption of straylight.

The origin of this local brightening still remains unclear. Bellot Rubio (2003) showed that it can be modelled without a temperature enhancement, simply combining a height-gradient of velocity with a micro-turbulence which increases with radial distance from the spot centre. We accordingly allowed our inversion to fit both, velocity gradients and a micro-turbulence constant with height but independent from the location in the spot: the inversion code did not return essential vertical velocities at the locations of the "bright rim". Hence, any explanation of the "bright rim" in terms of Doppler shifts should also hold in the absence of vertical velocity components at these locations.

For SIR-adapted straylight, we find a strong radial variation of the micro-turbulence decreasing from $1 \mathrm{~km} \mathrm{~s}^{-1}$ in the umbra (in agreement with Kneer 1972; and Stellmacher \& Wiehr 1970), to $0.2 \mathrm{~km}$ in the penumbra (where del Toro Iniesta et al. 1994 obtained $0.6 \mathrm{~km} \mathrm{~s}^{-1}$ ). For constant straylight level of $5 \%$, however, SIR returns a micro-turbulence that is almost equal in the umbra and the outer penumbra, but with a local minimum at radial distances where the bright rim occurs.

The results presented in this section show the large influence of the "straylight" assumption on the interpretation of the bright rim. The two inversions performed with constant and 

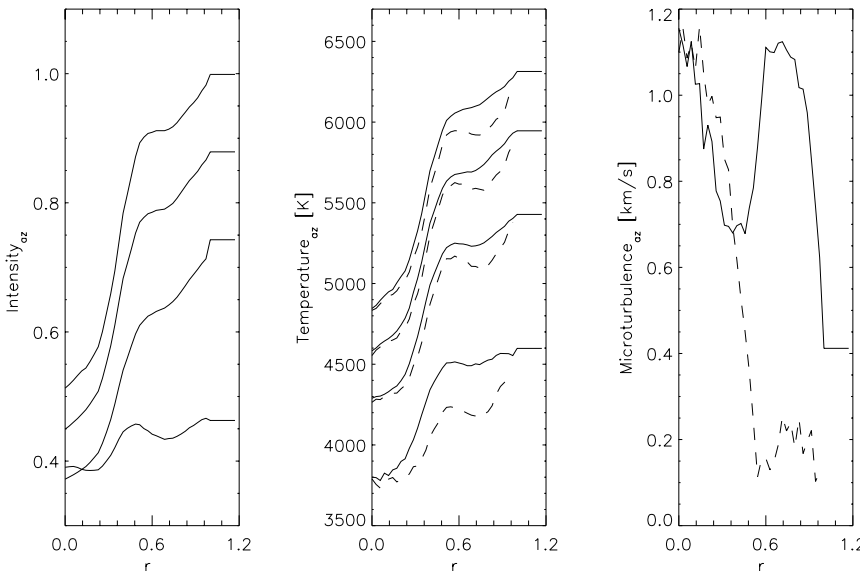

Fig. 6. Radial variation of azimuthally averaged intensity (left panel) in the continuum (upper curve), the line core (lower curve) and line positions in between, together with temperatures (middle panel) for $\log \tau=0$ (upper curve), $\log \tau=-0.5$ (upper middle curve), $\log \tau=-1$ (lower middle curve), $\log \tau=-2$ (lower curve), and micro-turbulence (right panel), as a function of the radial distance from the sunspot centre. Deduced quantities assuming a constant straylight factor of 5\% or free SIR-adopted straylight are denoted by solid lines and dashed lines, respectively.

with fitted straylight point to a thermal origin, but the height at which the heating occurs depends sensively on the straylight assumption. In Sects. 2.3 and 3.3 we present further indication that "straylight" is overestimated by SIR. We thus consider heating at high layers as the most probable source of the "bright rim".

\subsection{Flow configuration}

Doppler data depend sensively on a wavelength reference. The sunspot surroundings are not suitable as a velocity reference because of the granular blue shift ("limb effect"). Our field of view is not large enough to apply tabulated values of mean photospheric wavelengths. Also, magnetic structures are present in the spot surroundings, which significantly alter the granular velocity pattern (Balthasar et al. 1996). Thus, we assume the gas to be at rest in the darkest umbral core at the $\log \tau=0$ level (cf., Beckers \& Schröter 1969; Balthasar \& Schmidt 1993). This choice avoids unknown gas motions in umbral dots. Umbral oscillations that show peaks of $\pm 350 \mathrm{~m} \mathrm{~s}^{-1}$ (cf. Balthasar \& Wiehr 1984) yield an rms of $<150 \mathrm{~m} \mathrm{~s}^{-1}$ which is negligible in terms of our present study.

The obtained maps of LOS velocities (Fig. 7) show downflows that increase from $r / R_{\text {spot }}=0.6$ outwards to the spot border and beyond, reaching $3 \mathrm{~km} \mathrm{~s}^{-1}$ (in agreement with the values reported by Westendorp Plaza et al. 1997 in the spot surroundings). These vertical velocities occur predominantly at the lower layers $(\log \tau \geq-1.0)$. This height variation agrees with the one obtained by Schlichenmaier \& Schmidt (2000) and by Schlichenmaier et al. (2004), and fits the model of moving flux tubes of Schlichenmaier et al. (1998). However, it seems to disagree with the picture of "elevated Evershed

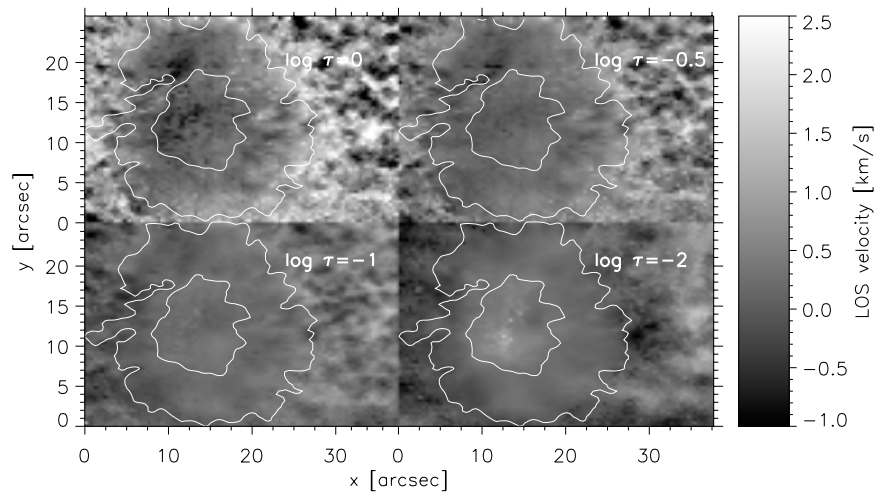

Fig. 7. Velocity maps of the disk centre spot at different layers; negative values denote upflows; the contours mark the outer boundary of umbra and penumbra.

channels" claimed by Rimmele (1995) and by Stanchfield et al. (1997).

The usual method to deduce the full velocity vector assumes axial flow symmetry. In this way, Schlichenmaier \& Schmidt (2000) found downflows through radial distances $r>$ $0.9 R_{\text {spot }}$, Tritschler et al. (2004) even at $r>0.7 R_{\text {spot, }}$, both increasing with radial distance. Velocities obtained under such an assumption of axial flow symmetry represent the mean azimuthally-averaged flow, where any departure from axial symmetry will result in a difficulty determining the velocity vector. Schlichenmaier \& Schmidt (2000) showed that a determination of the flow angle under such an assumption is particularly difficult at the spot border and beyond, where their deduced downflows become significant (although they are less than $500 \mathrm{~m} \mathrm{~s}^{-1}$ ).

Westendorp Plaza et al. (1997) obtained redshifts around the spot under study, exceeding $3 \mathrm{~km} \mathrm{~s}^{-1}$ at certain locations. They observed the spatial distribution of such "downflows" (i.e., in the observer reference frame, but not with respect to the solar surface) rather than azimuthal averages and confirmed that downflows take place in patches, not over axisymmetrical, uniformly distributed regions. In order to estimate the mass flux, the vertical velocity component was obtained under the assumption that the flow follows the magnetic field lines (as expected for the plasma condition in sunspots). However, one component inversions lead to flows having larger inclinations than the (mean) magnetic field (Klvana \& Bumba 1998); this will alter the obtained values of the downflows. With two-component inversions, Bellot Rubio et al. (2003) obtained flows that are aligned with a more horizontal field component than the background magnetic field. These authors assumed axial symmetry of the flow in order to obtain the full velocity vector for both the Evershed and the background magnetic components. They found downward flows of about $2 \mathrm{~km} \mathrm{~s}^{-1}$ for radial distances of $r>0.8 R_{\text {spot }}$, in better agreement with our results than any other velocity deduced under the assumption of axial symmetry.

Rimmele (1995) performed spectroscopic measurements in a $2 \mathrm{~h}$ time series and measured LOS velocities of $\sim 500 \mathrm{~m} \mathrm{~s}^{-1}$, which he considered to be downflows, since his spot was near the disk centre. However, during that time series, the spot 
changed its heliocentric angle to a maximum of as much as $10^{\circ}$ $(\cos \theta>0.985)$, being sufficiently far from the disk centre to obtain significant LOS velocity components from the horizontal Evershed flow (Solanki 2003).

A heliocentric angle of $2^{\circ}$, as in the present paper, can produce LOS velocities of about $200 \mathrm{~m} \mathrm{~s}^{-1}$ from a horizontal flow of $6 \mathrm{~km} \mathrm{~s}^{-1}$ due to projection effects, which by no means can explain the velocities observed. Hence, our maps presented in Fig. 7 confirm previous results of downflows at the outer penumbra (and beyond) for lower atmospheric layers; however, we obtain the vertical velocity component at much higher confidence without assuming axial symmetry of the flow.

We do not find significant flows in the inner penumbra where Rimmele (1995) found upflows of $200 \mathrm{~m} \mathrm{~s}^{-1}$, and Schlichenmaier et al. (2004) and Tritschler et al. (2004) found indirect evidence of small upflows. Westendorp Plaza et al. (2001b) observed blue-shifts in the inner to middle parts of the centre-side penumbra. Bello González (2004) found bright rising structures preferentially in the centre-side penumbra. Such upward motions in the inner penumbra might either be missed in our data due to the finite spatial resolution, or may stem from deeper layers than those sampled by the lines used here. However, we find local patches of upflows $\left(<1 \mathrm{~km} \mathrm{~s}^{-1}\right)$ in the outer middle penumbra. Schlichenmaier \& Solanki (2003) argued that several upflow channels are required to heat the entire penumbra by steady upflows along magnetic flux tubes. The inner penumbral upflow patches seen in Fig. 7 may then be the footpoints of the outer penumbral magnetic arches. Such a scenario also requires that significant downflows occur in the penumbra, as we find in Fig. 7.

Solanki \& Montavon (1993) and Martinez Pillet (2000) argued that their observations are only reproduced if flows are present along both the horizontal flux tubes carrying the Evershed flow and the steeper background magnetic field. The absence of significant $\delta A$ in the Stokes- $V$ in our observations and the retrieved velocity structure do not show any evidence for the existence of a significant flow along the background component.

A localised patch of upflows is found adjacent to the "perturbation" at $x \sim 0^{\prime \prime}-10^{\prime \prime}, y \sim 10^{\prime \prime}-15^{\prime \prime}$ presenting larger and more vertical magnetic fields. The nature of this upflow patch and its spatial correlation with the penumbral magnetic field perturbation remains to be determined.

In the umbra, we find a patch of red-shifts at $\log \tau=-2$ (i.e., $\sim 300 \mathrm{~km}$ above $\tau=0$ ), which could be interpreted as a signature of the chromospheric umbral downflow. Just outside the sunspot, we find local downflows associated with stronger and more vertical magnetic fields than in the more distant spot surroundings; these might be related to the flux concentrations in the spot moat (e.g. Wiehr et al. 2004).

In Fig. 8 we present the velocities as azimuthal averages along circular paths (cf. Fig. 4). As expected, these averaged vertical velocities are smaller than the local ones in Fig. 7. They show essentially zero velocities at all optical depths in the umbra and very small downflows up to $r / R_{\text {spot }} \sim 0.6$. For larger radial distances we find an increasing downflow in lower layers reaching $1.2 \mathrm{~km} \mathrm{~s}^{-1}$, while for higher layers the gas is essentially at rest. This result is independent of the
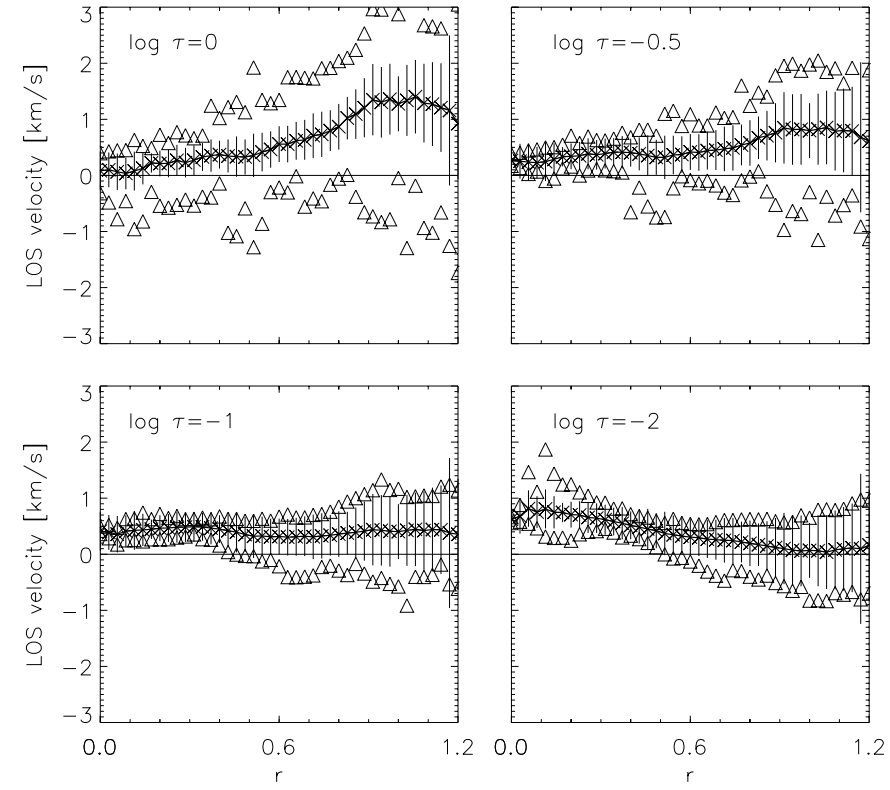

Fig. 8. Radial variation of azimuthally-averaged LOS velocities retrieved with 5\% constant straylight (solid-thick line) and SIR-adapted straylight (dashed-thin line) for different heights. Error bars represent the velocity standard deviations in each radial bin and triangles their extreme values.

assumption about straylight. The downflows span the outer half of the penumbra, in agreement with predictions from flux considerations by Schlichenmaier \& Solanki (2003).

\subsection{Magnetic configuration}

As already expected from the raw Stokes images (Fig. 1), the magnetic field is vertical near the umbral centre and turns gradually to the horizontal in the penumbra. This is confirmed by the magnetic field obtained with the SIR inversion (Fig. 9). For some locations at the penumbral border and beyond, the inclination exceeds $90^{\circ}$ in the deeper layers. Careful inspection reveals that these "return" field lines occur preferentially at spatial locations with abnormal 3-lobed Stokes- $V$ profiles. Since we observed the spot very close to the disk centre, the retrieved maps in Fig. 9 do not show the previously reported differences between limb-ward and disk-ward penumbral zones (Westendorp Plaza et al. 2001a; Mathew et al. 2003).

The magnetic field inclination is found to be largely independent of the height level. This contrasts with a variation of the field inclination by as much as $45^{\circ}$ in the range $0 \geq \log \tau \geq-1.5$ found by Mathew et al. (2003). According to Sánchez Almeida \& Lites (1992) such large vertical gradients of the mean magnetic field inclination do not reflect the real magnetic configuration of the sunspot but are artifacts being required to reproduce the observed $\delta A$. We consider the vertical gradient of the magnetic field inclination obtained in the present work to be more realistic.

We find a tight relation between the strength and the inclination of the sunspot magnetic field, in agreement with previous works (Hale \& Nicholson 1938; Beckers \& Schröter 1969) but in disagreement with the findings by Solanki et al. (1992). 

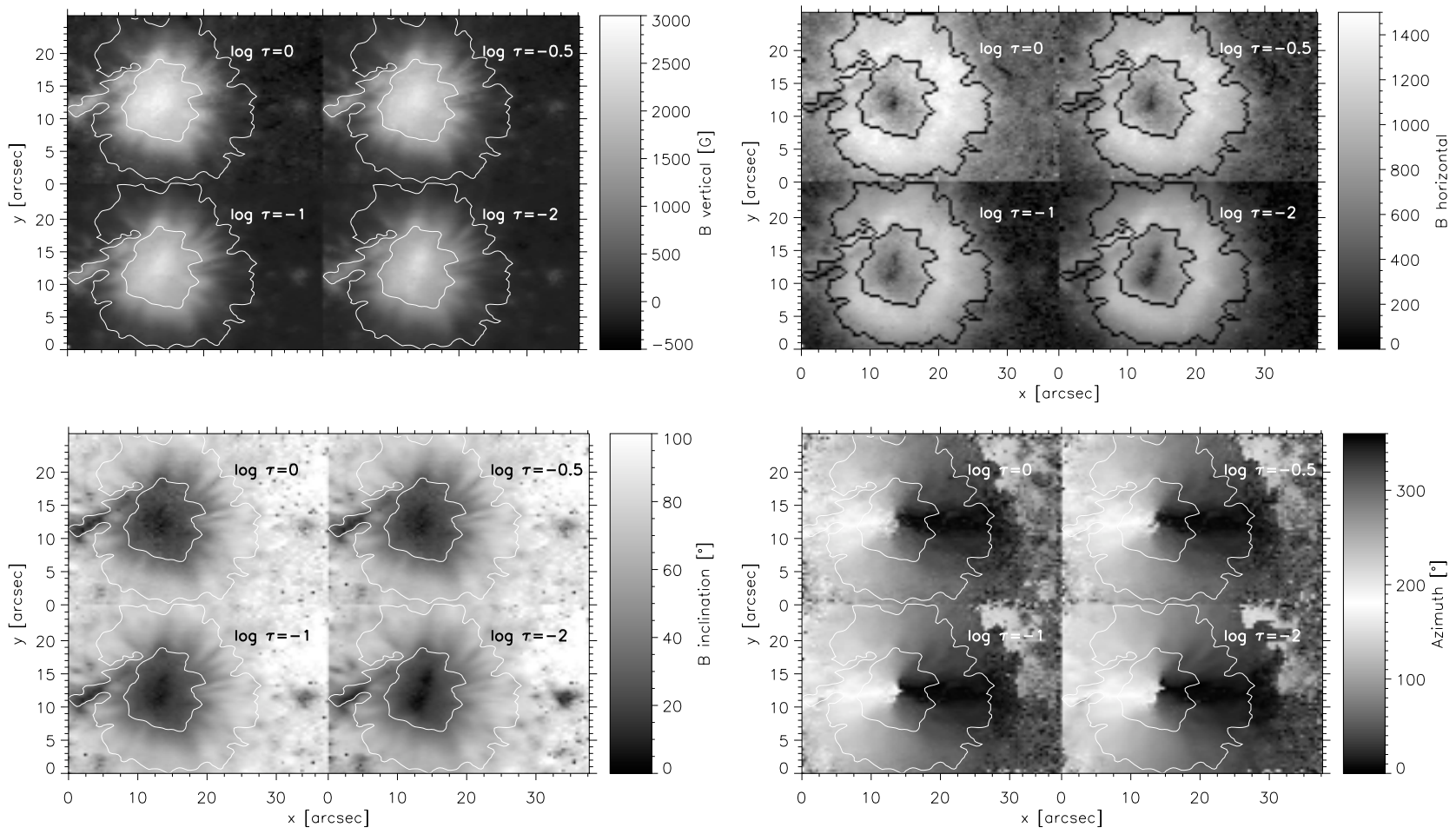

Fig. 9. Maps of the vertical (upper) and the horizontal (second) components of the magnetic field vector, its inclination (third) and its azimuth (lower panel) at 4 different atmospheric height levels marked in each sub-panel. The contours mark the outer boundary of the umbra and of the penumbra.
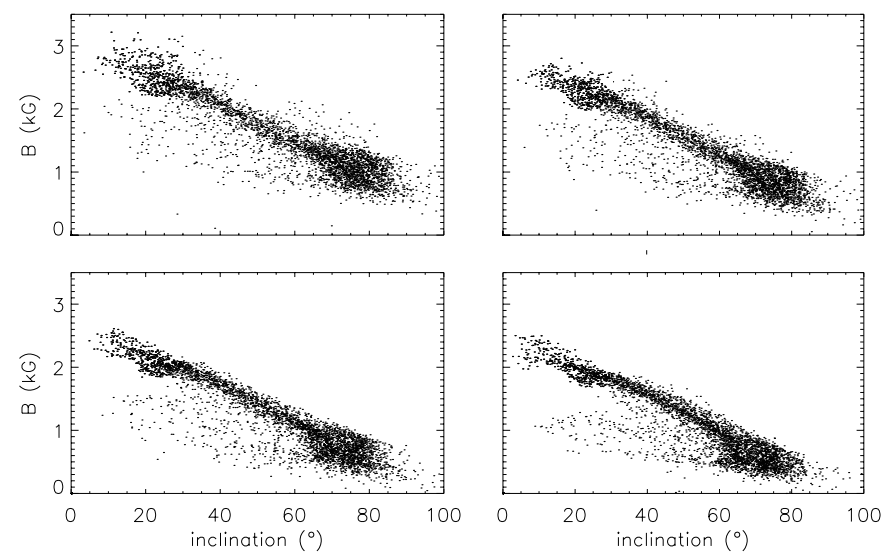

Fig. 10. Scatter-plots of strength and inclination of the magnetic field for the height levels $\log \tau=0$ (upper left), $\log \tau=-0.5$ (upper right), $\log \tau=-1.0$ (lower left), and $\log \tau=-2$ (lower right).

The scatter-plots in Fig. 10 show that stronger fields are steeper with a distinct gradient of $35^{\circ} / 1000 \mathrm{G}$, largely independent of height. This gradient is significantly flatter than those found by Westendorp Plaza et al. (2001a) and by Mathew et al. (2004) who obtained as much as $50^{\circ} / 1000 \mathrm{G}$ and not a clear independence of the height. Their larger gradients might originate from projection effects from the oblique LOS.

The scatter-plots of Fig. 10 indicate a bifurcation at the high layers. Westendorp Plaza et al. (2001a), however, found such a bifurcation at the lower layers, and interpret it as a signature of the penumbral fluting. Close inspection of our results shows that the data points forming the second branch stem from the umbral-penumbral boundary, as well as from the umbral "extension" at $x \sim 0$ " $-10^{\prime \prime}, y \sim 10^{\prime \prime}-15^{\prime \prime}$ in Fig. 9. Both show larger and more vertical magnetic fields (associated with local brightenings of the line cores) than in the surrounding penumbra.

The azimuth of the magnetic field indicates that a deviation from the purely radial direction is less than $5^{\circ}$ at all height levels (Fig. 9); this is most impressively seen in the azimuthal averages Fig. 11. Only at the sunspot centre are larger deviations found which can be attributed to the small number of pixels involved in the central averaging ring. Hence, we do not find any indication of a field vortex, - neither with radial distance nor with height. Lites \& Skumanich (1990), Skumanich et al. (1994), Keppens \& Martínez Pillet (1996) and Westendorp Plaza et al. (2001a) found larger residual twists of up to $15^{\circ}$.

Although we only observe the immediate surroundings of the sunspot, the obtained azimuths seem not to coincide with the vortexes observed in the corresponding $\mathrm{H} \alpha$ image (Fig. 12). Kawakami et al. (1989) found near disk centre a coincidence rate of about $50 \%-70 \%$ of the transverse photospheric magnetic field and the $\mathrm{H} \alpha$ fibrils.

In the spot surroundings, we obtain small horizontal magnetic fields which decrease with height. This contrasts with Westendorp Plaza et al. (2001a) who found an increasing field strength with height, which they interpreted as a signature of the "canopy".

We find that the canopy is pierced by more vertical "plage" magnetic fields associated with down-flows which might be identified as the foot-points of magnetic arches hosting a flow 

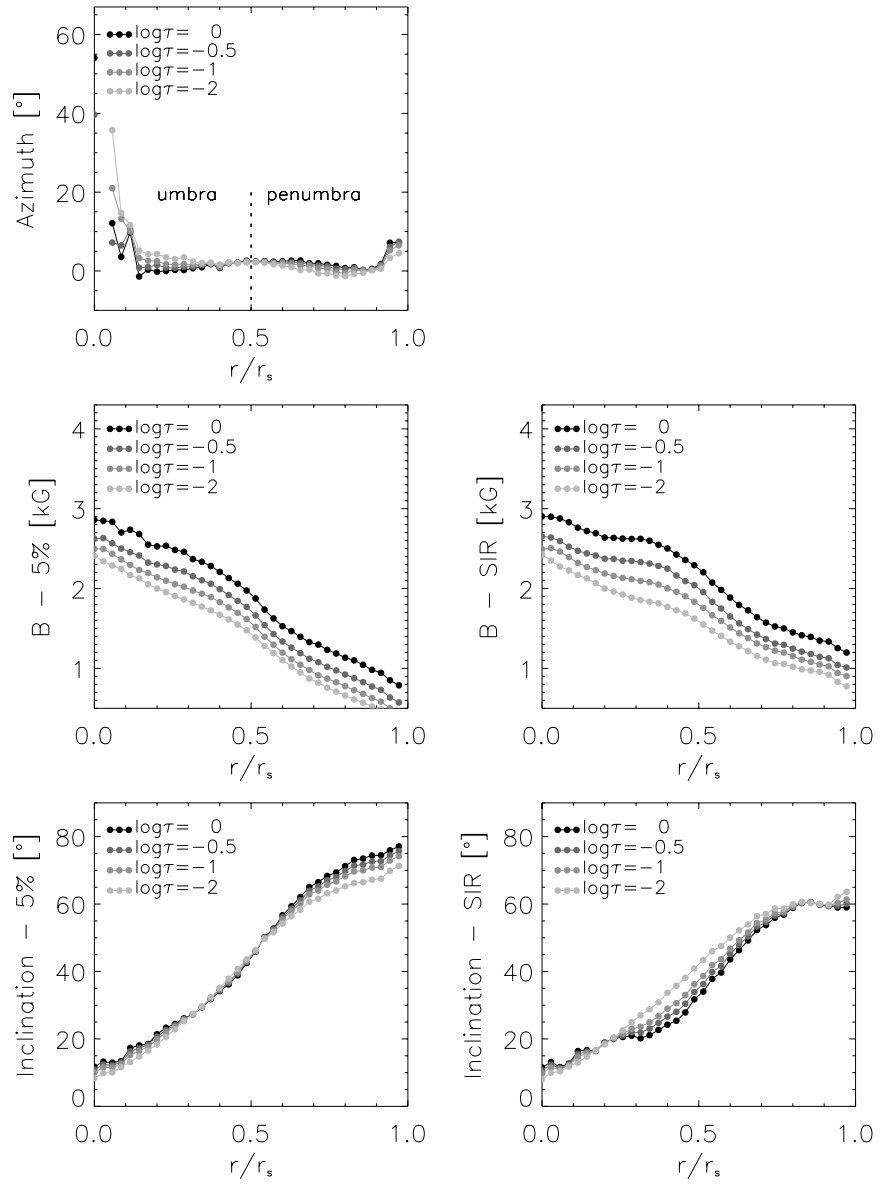

Fig. 11. Radial variations of the azimuthally-averaged field azimuth, field strength and field inclination; the latter two are presented for 5\% constant straylight and for SIR-adapted straylight. Each subfigure contains 4 height levels.

(e.g., Börner \& Kneer 1992), driven by the pressure difference between the two foot-points (as suggested by the siphon flow model by Meyer \& Schmidt 1968). However, our finding of strong down-flows in the outer penumbra and its near surroundings (cf. previous section) favours the return flux model proposed by Weiss et al. (2004), in which downward pumping of the magnetic flux causes a submergence of field lines in the immediate surroundings of the sunspot, where the convective energy exceeds the magnetic one (cf. Wiehr 1996).

The radial variations of azimuthal averages in Fig. 11 show that the magnetic field strength decreases with height and with radial distance; this conflicts with Westendorp Plaza et al. (2001a), who found for the outer penumbra larger magnetic field strengths at high layers. The slope of their observed decrease slightly changes at $r=0.6 R_{\text {spot }}$, in agreement with Bellot Rubio (2003) who found a change near $r=0.7 R_{\text {spot }}$. Our two alternative assumptions for straylight show almost the same height dependence of the field, but a slightly flatter radial variation for SIR-adapted straylight (here, SIR assumes most of the Stokes- $I$ profile to be of photospheric, i.e., hot origin, the remaining penumbral atmosphere then being colder; lower temperatures produce smaller amplitudes of, particularly,

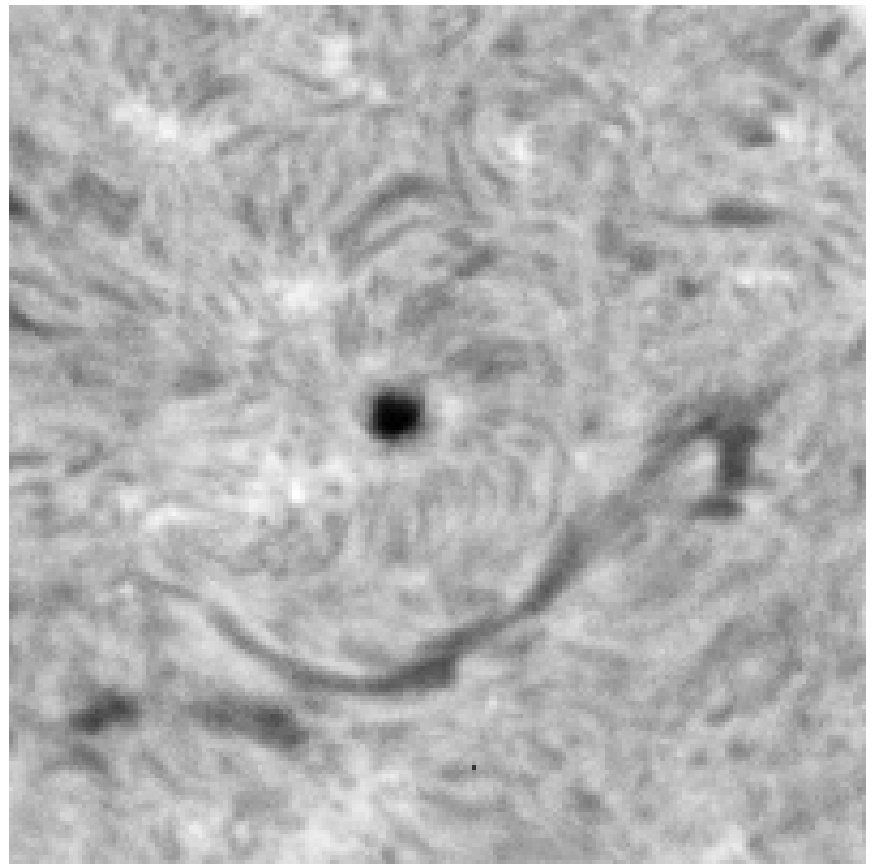

Fig. 12. The sunspot from Sep. 23, 2003, at disk centre in $\mathrm{H} \alpha$ (courtesy A. Tritschler, NSO/Sac Peak), showing some indication of vortex structures in the super-penumbra.

Stokes- $V$; for an optimal fit, the SIR code is forced to increase the magnetic field strength).

The azimuthally-averaged field inclination increases radially; its mean does not reach values larger than $90^{\circ}$, i.e. no oppositely-directed magnetic fields are observed. The difference in the obtained inclinations for constant and for SIR-adapted straylight may well be due to the fact that the retrieved field strength also differs between those two assumptions (see Fig. 11). Stenflo (1985) showed that an underestimation of the magnetic field strength yields too large values for the field inclination. Recent investigations give overwhelming evidence for inclinations of $70^{\circ}-80^{\circ}$ at the spot border (Title et al. 1993; Skumanich et al. 1994; Keppens \& Martinez Pillet 1996; Westendorp Plaza et al. 2001a); this is in better agreement with the values we obtained with constant $(5 \%)$ rather than with SIR-adapted straylight (Fig. 11). Indeed, Solanki (2003) argued that earlier results of inclinations near $90^{\circ}$ (Beckers \& Schröter 1969; Wittman 1974; Giovanelli 1982) were due to an insufficient correction for straylight; this points to an overcorrection if the straylight is adapted by SIR as a free parameter.

\subsection{Azimuthal variations}

The round shape of the sunspot allows the study of azimuthal scans along selected circular paths (Fig. 4). Although the spatial resolution is in this data far beyond that required to resolve the penumbral filamentary structure, our observations do resolve "conglomerates" of penumbral structures visible as "spines" in Fig. 1. The azimuthal scans (Fig. 13) show that for all observed heights in the penumbra, stronger magnetic fields are more vertical, in agreement with Degenhardt \& Wiehr (1991), Lites et al. (1993) and Stanchfield et al. (1997), but 

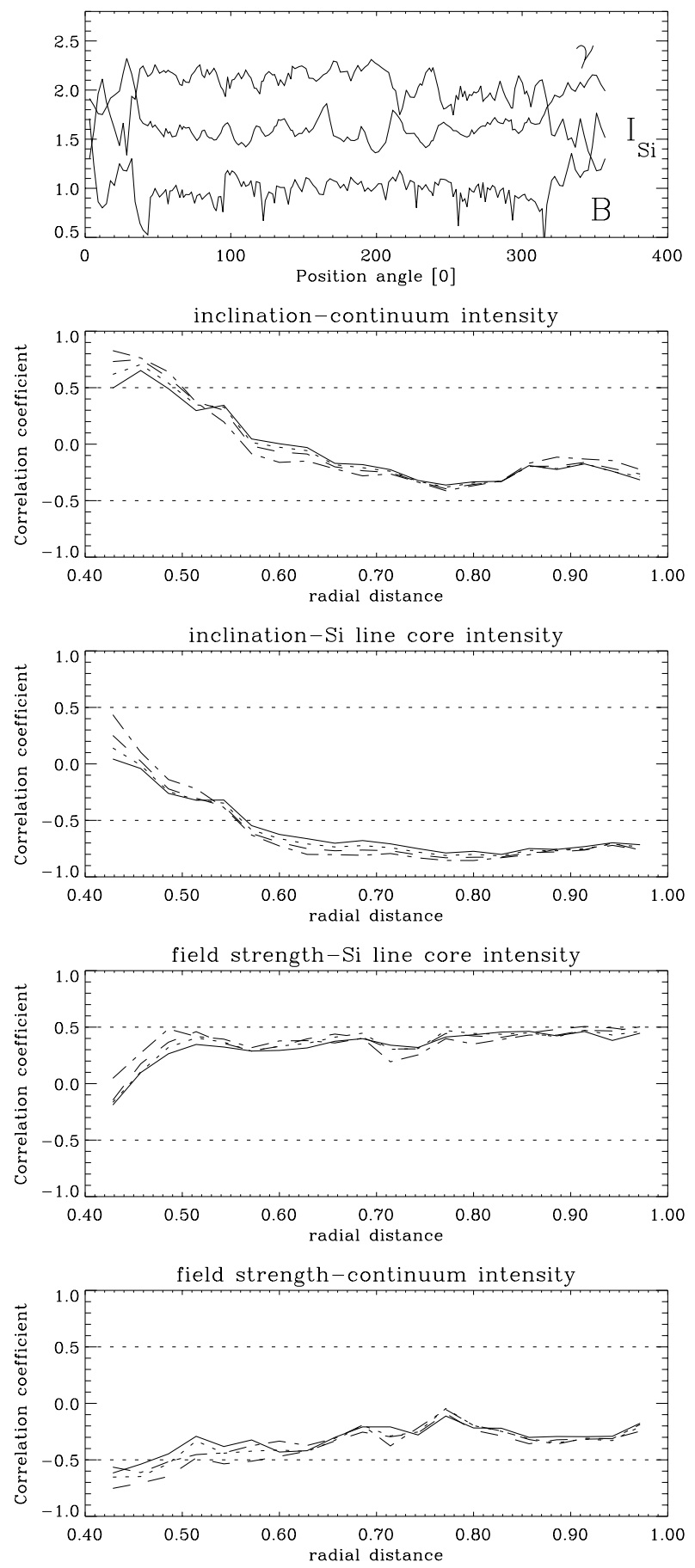

Fig. 13. Uppermost panel: azimuthal variation of field inclination (upper curve), line core intensity of Si 10787 (middle curve) and field strength (lower curve) along an azimuthal path at a radial distance of $r=0.8$ at $\log \tau=0$; lower panels: radial variation of the corresponding correlation coefficients for $\log \tau=0$ (solid line), -0.5 (dotted), -1.0 (dashed), -2 (dashed-dotted); the umbral-penumbral transition is located at $r=0.4-0.5$.

in disagreement with Wiehr (2000). Westendorp Plaza et al. (2001a) found a negative correlation of the magnetic field strength and inclination in the mid penumbra for $\log \tau=-1.5$, which turns into a positive correlation at the outer sunspot boundary at lower atmospheric layers. This contradicts our finding of a height-independent negative correlation. When evaluating the different results, one has to consider that they are deduced from rather different lines which may (at least partly) be responsible for the discrepancies (Wiehr 2000). Greater steepness and stronger fields just compensate each other in the projection on the solar surface, thus causing no fluctuation of the radial magnetic field component along the azimuthal paths. In contrast, the vertical component shows more pronounced "spines" (Fig. 9); they are already visible in the raw Stokes- $V$ image (Fig. 1).

We find no relation of the magnetic field with the continuum intensity, in agreement with Hirzberger \& Kneer (2001), but we do see a significant relation with the line core intensities. Flatter and fainter fields are preferentially related to reduced intensity (Fig. 13). This cannot be explained by the splitting of the Si I $10787 \AA$ line which indeed produces higher line core intensities for steeper fields. However, simple test calculations show that only $15 \%$ of the observed line core brightenings can be assigned to this effect.

The finding that the correlation of the magnetic field with the intensity line cores from high layers is larger than that with the continuum intensity suggests that the steeper and stronger fields represent the "background component"; the flatter and fainter field component might then host the Evershed flow. We find no significant correlation of the velocity with the strength or the inclination of the magnetic field; this agrees with Wiehr \& Degenhardt (1984) who showed that such a relation can only be observed if the spectra are spatially very high resolved, which is not the case for the present data. Bellot Rubio et al. (2004) found from a two-component inversion that the flat component hosts more horizontal and weaker magnetic fields than the steep background component. They found the filling factor of these components to fluctuate azimuthally, suggesting that dark penumbral "spines" are mostly a change of the "visibility" of the flatter field component.

\section{Summary and conclusions}

A sunspot at solar disk centre $\left(\theta=2^{\circ}\right)$ was observed by means of the full Stokes spectra of three infrared lines. The thermal, flow and magnetic configurations are determined. The lack of differential optical or projection effects yields a symmetrical distribution of these quantities, without the previously reported differences between a centre- and a limb-side penumbra. The spectra show regular double-lobed Stokes- $V$ profiles throughout the spot with net area asymmetries $\delta A<1 \%$. Thus, a single magnetic component inversion is especially suitable to characterise the "mean" magnetic, flow and thermal configuration, since no artifacts (such as artificially large gradients of the magnetic field inclination) are produced for a fit of the asymmetric Stokes- $V$ profiles often observed at larger heliocentric angles.

The temperature is not found to increase monotonically with radial distance from the centre of the spot, but shows an enhancement in the inner penumbra. The height level at which this "hump" occurs depends on the assumption for the straylight correction. We find indication of an over-estimation of "straylight" when adapted by the SIR code. By setting the straylight to an estimated maximum of $5 \%$, constant over the 
spot, we find the temperature enhancement to be restricted to higher layers.

Due to the disk centre position of the spot, the vertical velocity components of the flow vector are directly measured as LOS velocities. We find a patchy downflow distribution with velocities which increase from the $r / R_{\text {spot }}=0.6$ to the outer penumbral boundary, reaching $3 \mathrm{~km} \mathrm{~s}^{-1}$ at distinct spatial locations. Downflows are mainly found at the deepest layers, disappearing higher up in the atmosphere. Such a flow configuration agrees with the picture of low lying horizontal fields that host the Evershed flow (Schlichenmaier et al. 1998).

The typical signature of the Evershed effect, the pronounced line asymmetry, is known to disappear abruptly at the outer edge of the white light penumbra (Balthasar \& Wiehr 1989; Hirzberger \& Kneer 2001). Our finding of downward velocities even outside that border apparently disagree with the reported spatial behaviour of the line asymmetry. Solanki et al. (1994) reported the Evershed effect to continue outside the white light boundary of the sunspot only above the canopy base height, i.e. in the upper photosphere. If the downflows detected in the present paper were related to the Evershed flow, than it should continue well outside the sunspot boundary in deep layers. However, the local flow and field patches outside the penumbra could also be a signature of local magnetic fields in the sunspot moat (Wiehr et al. 2004).

Although our single-component inversion cannot confirm distinct field components, these are indicated by the finding that steeper and stronger magnetic fields are related to intensity brightenings in the line cores but not in the continuum. This favours the assumption that at lower layers the presence of at least two different magnetic components inhibits a significant correlation with the continuum intensity, whereas at upper layers a background component dominates which leads to a correlation with the line core intensity. Our results support that the magnetic component hosting the Evershed flow is weaker and flatter and dives back to the solar surface at the penumbra and beyond, in agreement with Bellot Rubio et al. (2004). The stronger and steeper background component may extend far beyond the white light spot edge towards higher layers, finally forming the superpenumbra.

In the sunspot, a magnetic field decreasing smoothly with radial distance and with height is obtained, in contradiction to some previous reports (Westendorp Plaza et al. 2001a). At the outer spot edge, the field strength decreases to values small enough for a dominance of the convective energy over the magnetic one in agreement with Wiehr (1996). The magnetic field inclination is found to be largely independent on height and shows a tight relation to the field strength with $35^{\circ} / 1000 \mathrm{G}$. The inclination at the outer sunspot boundary depends on the assumption used for straylight correction, ranging from $60^{\circ}$ to $80^{\circ}$.

We find a highly radial field azimuth without any indication of vortices at all height levels covered by our lines. This result conflicts with the field vortices observed in the $\mathrm{H} \alpha$ superpenumbra far outside the white light boundary. Such vortices likely do not arise from Coriolis forces, since they often exhibit the opposite direction of the flow at different locations in one penumbra (Pevtsov et al. 2003). The superpenumbral structures may be a signature of the steep background magnetic component, connecting the spot to the corresponding magnetic region with opposite polarity.

Acknowledgements. We thank Drs. M. Collados, B. Ruiz Cobo and H. Balthasar for fruitful discussions and suggestions. We thank the referee, Dr. M. Penn, for suggestions and comments which significantly improved this paper. This research is part of the TMR-ESMN (European Solar Magnetism Network) supported by the European Commission under contract HPRN-CT-2002-00313. K.G.P. obtained support by the "Deutsche Forschungsgemeinschaft" through grand KN 152/29-1. The Vacuum Tower Telescope on Tenerife is operated by the Kiepenheuer-Institut für Sonnenphysik (Germany) at the Spanish "Observatorio del Teide" of the Instituto de Astrofísica de Canarias.

\section{References}

Balasubramaniam, K. S. 2002, ApJ, 575, 553

Balthasar, H., \& Wiehr, E. 1984, Sol. Phys., 94, 99

Balthasar, H., \& Wiehr, E. 1989, A\&A, 208, 303

Balthasar, H., \& Schmidt, W. 1993, A\&A, 279, 243

Balthasar, H., Schleicher, H., Bendlin, C., \& Volkmer, R. 1996, A\&A, 315,603

Beckers, J. M., \& Schröter, E. H. 1969, Sol. Phys., 10, 384

Bello González, N., Okunev, O. V., Domínguez Cerdeña, I., Kneer, F., \& Puschmann, K. G. 2004, A\&A, submitted

Bellot Rubio, L. R., Balthasar, H., Collados, M., \& Schlichenmaier, R. 2003, A\&A, 403, L47

Bellot Rubio, L. R. 2003, in 3rd International Workshop on Solar Polarization, ed. J. Trujillo Bueno, \& J. Sánchez Almeida, ASP Conf. Ser., 307, 301

Bellot Rubio, L. R., Balthasar, H., \& Collados, M. 2004, A\&A, 427, 319

Bernasconi, P. N., Keller, C. U., Solanki, S. K., \& Stenflo, J. O. 1998, A\&A, 329, 704

Borrero, J. M., Solanki, S. K., Bellot Rubio, L. R., Lagg, A., \& Mathew, S. K. 2004, A\&A, 422, 1093

Börner, P., \& Kneer, F. 1992, A\&A, 259, 307

Collados, M., Martínez Pillet, V., Ruiz Cobo, B., del Toro Iniesta, J. C., \& Vázquez, M. 1994, A\&A, 291, 622

Degenhardt, D., \& Wiehr, E. 1991, A\&A, 252, 821

Degenhardt, D., \& Lites, B. W. 1993, ApJ, 416, 875

del Toro Iniesta, J. C., Tarbell, T. D., \& Ruiz Cobo, B. 1994, ApJ, 436, 400

Giovanelli, R. G. 1982, Sol. Phys., 82, 21

Hale, G. E., \& Nicholson, S. B. 1938, Washington, D.C. Carnegie Institution of Washington, 1938

Hewagama, T., Deming, D., Jennings, D. E., et al. 1993, ApJS, 86, 313

Hirzberger, J., \& Kneer, F. 2001, A\&A, 378, 1078

Kawakami, S., Makita, M., \& Kurokawa, H. 1989, Astron. Soc. Japan, 41,175

Keppens, R., \& Martínez Pillet, V. 1996, A\&A, 316, 229

Klvana, M., \& Bumba, V. 1998, ASP Conf. Ser., 155, 79

Kneer, F. 1972, A\&A, 18, 39

Leka, K. D. 2001, 20th NSO/Sac Summer Workshop, ASP Conf. Proc., 236, 571

Lites, B. W., \& Schumanich, A. 1990, ApJ, 348, 747

Lites, B. W., Elmore, D. F., Seagraves, P., \& Skumanich, A. P. 1993, ApJ, 418, 928 
Martínez Pillet, V. 1992, Sol. Phys., 140, 207

Martínez Pillet, V., Collados, M., Bellot Rubio, L. R., et al. 1999, AG Abstract Services, Vol. 15. Abstracts of the Annual Scientific Meeting of the Astronomische Gesellschaft, in Göttingen, 20-25 September 1999

Martínez Pillet, V. 2000, A\&A, 361, 734

Mathew, S. K., Lagg, A., Solanki, S. K., et al. 2003, A\&A, 410, 695

Mathew, S. K., Solanki, S. K., Lagg, A., et al. 2004, A\&A, 422, 693

Meyer, F., \& Schmidt, H. U. 1968, Mitteilungen der Astronomischen Gesellschaft, 25, 194

Pevtsov, A. A., Balasubramaniam, K. S., \& Rogers, W. J. 2003, AdSpR, 32(10), 1905

Rimmele, T. R. 1995, ApJ, 445, 511

Ruiz Cobo, B., \& del Toro Iniesta, J. C. 1992, ApJ, 389, 375

Sánchez Almeida, J., \& Lites, B. W. 1992, ApJ, 398, 359

Sánchez Almeida, J. 1998, ApJ, 497, 967

Schlichenmaier, R., Jahn, K., \& Schmidt, H. U. 1998, A\&A, 337, 897

Schlichenmaier, R., \& Schmidt, W. 2000, A\&A, 358, 1122

Schlichenmaier, R., \& Collados, M. 2002, A\&A, 381, 668

Schlichenmaier, R., Bellot Rubio, L. R., \& Tritschler, A. 2004, A\&A, 415,731

Schlichenmaier, R., Bellot Rubio, L. R., \& Tritschler, A. 2005, AN, 326(3), 301

Schlichenmaier, R., \& Solanki, S. K. 2003, A\&A, 411, 257

Schmidt, W., \& Balthasar, H. 1994, A\&A, 283, 241

Schmidt, W., \& Fritz, G. 2004, A\&A, 421, 735

Skumanich, A., Lites, B. W., \& Martínez Pillet, V. 1994, in Solar Surface Magnetism, ed. R. J. Rutten, \& C. J. Schrijver (Dordecht: Kluwer), 99

Solanki, S. K. 2003, A\&AR, 11, 153

Solanki, S. K., Rüedi, I., \& Livingston, W. 1992, A\&A, 263, 339

Solanki, S. K., \& Montavon, C. A. P. 1993, A\&A, 275, 283
Solanki, S. K., Montavon, C. A. P., \& Livingston, W. 1994, A\&A, 283, 221

Stanchfield, D. C. H., Thomas, J. H., \& Lites, B. W. 1997, ApJ, 477, 485

Stellmacher, G., \& Wiehr, E. 1970, A\&A, 7, 432

Stellmacher, G., \& Wiehr, E. 1971, Sol. Phys., 71, 21

Stellmacher, G., \& Wiehr, E. 1980, A\&A, 82, 157

Stellmacher, G., \& Wiehr, E. 1981, A\&A, 95, 229

Stenflo, J. O. 1985, Sol. Phys., 100, 189

Title, A. M., Frank, Z. A., Shine, R. A., et al. 1993, ApJ, 403, 780

Tritschler, A., Schlichenmaier, R., Bellot Rubio, L. R., \& the KAOS Team 2004, A\&A, 415, 717

Weiss, N. O., Thomas, J. H., Brummell, N. H., \& Tobias, S. M. 2004, ApJ, 600, 1073

Westendorp Plaza, C., del Toro Iniesta, J. C., Ruiz Cobo, B., et al. 1997, ASP Conf. Ser., 118, 202

Westendorp Plaza, C., del Toro Iniesta, J. C., Ruiz Cobo, B., et al. 2001, ApJ, 547, 1130

Westendorp Plaza, C., del Toro Iniesta, J. C., Ruiz Cobo, B., \& Martínez Pillet, V. 2001, ApJ, 547, 1148

Wiehr, E., \& Degenhardt, D. 1992, A\&A, 265, 781

Wiehr, E., Koch, A., Knölker, M., Küveler, G., \& Stellmacher, G. 1984, A\&A, 140, 352

Wiehr, E., \& Stellmacher, G. 1984, IAU-Proc. of the 8-th Europ. Reg. Astr. Meeting, Toulouse, 254

Wiehr, E. 1996, A\&A, 309, L4

Wiehr, E. 2000, in Magnetic Fields an Oscillations, ed. B. Schmieder, A. Hofmann, \& J. Staude, 3rd Adv. Sol. Phys. Euroconf., ASP Conf. Ser., 184, 86

Wiehr, E., Bovelet, B., \& Hirzberger, J. 2004, A\&A, 422, L63

Wittmann, A. 1974, Sol. Phys., 36, 29

Wöhl, H., Wittmann, A., \& Schröter, E. H. 1970, Sol. Phys., 13, 104 